\title{
Níveis Econômicos de Prejuízos de Plantas Infestantes nas Culturas Agrícolas: Conceitos, Definições e Formas de CÁlCUlo ${ }^{1}$
}

\author{
Economic Levels of Weed Injury on Crops: Concepts, Definitions and Calculation Models
}

PORTUGAL, J.M. ${ }^{2}$ e VIDAL, R.A. ${ }^{3}$

\begin{abstract}
RESUMO - Uma das áreas da matologia (herbologia em Portugal) que tem avançado nas pesquisas científicas em Portugal e no Brasil nas últimas três décadas é a de nivel de prejuizo das infestantes às culturas agrícolas. Contudo, durante esse periodo ocorreu uma multiplicidade de traduções de termos técnicos para a lingua portuguesa, que atualmente dificultam o intercâmbio de informações técnico-científicas. Os objetivos desta revisão de literatura foram: definir os cinco diferentes termos referentes aos niveis de prejuizos das infestantes utilizados em herbologia; consolidar no idioma português os acrônimos utilizados para esses termos; apresentar as designações que têm correspondência nas linguas francesa e inglesa; indicar as perguntas científicas a que o termo responde; e apresentar as formas de cálculo que podem ser utilizadas para cada nivel de prejuizo definido. Os termos estudados nesta revisão foram: densidade critica, nível prejudicial de ataque (NPA ou nível de dano econômico $=\mathrm{NDE}$ ), nivel econômico de ataque, nivel de tolerância e nivel de segurança de ataque. Os modelos de cálculo do NPA = NDE no caso de infestações contendo uma única espécie vegetal são deduzidos a partir dos modelos linear, hiperbólico, múltiplo linear e múltiplo não linear; e para as situações de infestações com mais de uma espécie vegetal, a partir da equação linear múltipla entre a densidade de plantas e o rendimento da cultura.
\end{abstract}

Palavras-chave: equações, densidade crítica, nível prejudicial, nível de dano econômico, nível de tolerância, nível de segurança.

\begin{abstract}
Weed threshold levels of injury to agricultural crops is a branch of weed science that has presented advanced scientific research in Portugal and Brazil in the last three decades. However, during this period there has been a multitude of translations of technical terms from the English or French into Portuguese that have currently hindered the exchange of technical scientific information. The objectives of this literature review were to define the terms relating to five different levels of weed injury used in weed science; to consolidate the acronyms used for these terms in the Portuguese language, to provide the names that have correspondence in French and English, to indicate the scientific questions that the terms imply, and to present the forms of calculation that can be used for each level defined. The terms studied in this review were: critical density, economic injury level, economic threshold, level of tolerance, and level of security attack. The forms of calculation of economic threshold in the case of infestation with a single plant species are derived from linear, hyperbolic, multiple linear and multiple non-linear models; for cases of infestation involving more than one plant species, these forms are derived from multiple linear equation between weed density and crop yield.
\end{abstract}

Keywords: equations, critical density, economic injury level, economic threshold, tolerance level, safety level.

1 Recebido para publicação em 29.7.2009 e na forma revisada em 13.11.2009.

2 Engo-Agrō, Dr., Professor do Instituto Politécnico de Beja, Rua Pedro Soares 7800 - 295 Beja, Alentejo, Portugal, $<$ jportugal@ipbeja.pt>; ${ }^{3}$ Eng $^{0}$-Agr ${ }^{0}$, Dr., Professor da Universidade Federal do Rio Grande do Sul, Faculdade de Agronomia, Caixa Postal 15100, 90001-970, Porto Alegre-RS, Brasil, <ribas.vidal@ufrgs.br>.

Planta Daninha, Viçosa-MG, v. 27, n. 4, p. 869-877, 2009 


\section{INTRODUÇÃO}

A introdução da proteção integrada como modelo científico de estudo e gestão de infestantes nas culturas agrícolas acarretou, entre outros aspectos, a adoção de nova terminologia na matologia (herbologia em Portugal). Nesse contexto, destaca-se a introdução das designações relativas aos niveis de prejuízo, os quais constituem os fundamentos básicos da proteção integrada. Desde que começaram a ser utilizados, primeiramente para o manejo de insetos $\mathrm{e}$, posteriormente, para as infestantes, foram sendo definidos diferentes termos e conceitos de níveis de prejuízo. Alguns dos termos/conceitos apresentam objetivos semelhantes entre as áreas de entomologia e herbologia, mas outros não são iguais nem nos seus propósitos nem na forma como poderiam ser determinados ou empregados. Ao longo do tempo têm surgido múltiplas definições em várias línguas (e até na mesma língua) nessa área. Em alguns casos, os niveis são esclarecedores relativamente ao que se pretende deles, ou seja, são autoexplicativos. Entretanto, existem termos que contêm nuances ou sutilezas que nem sempre estão convenientemente esclarecidas, existindo até, por vezes, sobreposição de conceitos. Essa situação dá origem, ou poderá dar origem, a interpretações diferentes por parte daqueles que fazem seu uso, justificando que se harmonizem os termos empregados entre os diferentes idiomas e áreas do conhecimento (Fernandes, 1994, 2003).

Nos países de lingua portuguesa, ao longo das últimas três décadas têm-se ampliado os trabalhos científicos na área da determinação de niveis de prejuízo na herbologia. Merecem destaque as pesquisas realizadas em Portugal (Godinho \& Costa, 1980; Bento et al., 1987; Aguiar, 1992; Fernandes, 1994, 2003) e no Brasil (Fleck, 1996; Vidal et al., 1999, 2004; Theisen et al., 2000; Fleck et al., 2002; Passini et al., 2003; Rizzardi et al., 2003, 2004; Agostinetto et al., 2005; Bianchi et al., 2006; Galon et al., 2007). Contudo, nem sempre existe a correspondência entre as terminologias utilizadas nesses países.

O idioma português é utilizado por mais de 250 milhões de cidadãos dos cinco continentes e ocorrem diferenças de nomenclatura que, às vezes, dificultam a partilha do conhecimento científico. No ano de 2009 ocorre a harmonização da ortografia lusófona na literatura, o que justifica um esforço para uniformizar a terminologia no idioma português nas diferentes comunidades científicas.

Os objetivos desta revisão de literatura foram: definir os cinco diferentes termos referentes aos niveis de prejuízos das infestantes utilizados em herbologia; consolidar no idioma português os acrônimos utilizados para esses termos; apresentar as designações que têm correspondência nas línguas francesa e inglesa; indicar a(s) pergunta(s) científica(s) a que o termo responde; e apresentar as formas de cálculo que podem ser usadas para cada nível de prejuízo definido.

\section{INFESTAÇÃO COM PLANTAS DE UMA ÚNICA ESPÉCIE VEGETAL}

Neste item são apresentados individualmente os cinco tipos de nivel de prejuizo, começando pela designação a adotar para cada nível e o respectivo acrônimo. Apresentam-se as designações de cada nivel nos idiomas francês e inglês, com a finalidade de facilitar as revisões de literatura para a comunidade científica. Posteriormente, é apresentada a definição ou o conceito do nivel e, para os níveis mais importantes, aponta(m)-se a(s) pergunta(s) a que o nível responde e a(s) sua(s) forma(s) de cálculo.

a. Densidade critica. Também denominada Nivel Crítico de Ataque (NCA) em Portugal (Fernandes, 2003); na lingua francesa, densité critique (Caussanel et al., 1986); e na lingua inglesa, critical density (Cousens, 1988; Coble \& Mortensen, 1992). Corresponde ao nivel de infestação a partir do qual é mensurável, por testes de inferência, o efeito depressivo das infestantes no rendimento da cultura (Caussanel et al., 1986; Coussans et al., 1986). Com esse nível de prejuízo pretende-se responder à seguinte questão:

\section{- A partir de qual densidade de infestação se verifica redução significativa na produção?}

Normalmente a densidade de plantas daninhas não é constante durante todo o ciclo de vida da cultura. Assim, um componente temporal define o período crítico de ataque, chamado de periode critique e critical period 
threshold nos idiomas francês e inglês, respectivamente. Nesse caso, objetiva-se questionar:

\section{- Em que fase(s) do ciclo da cultura a interferência é significativa?}

As respostas a esta questão correspondem em herbologia aos conceitos de densidade crítica e período crítico, cujo cálculo se faz após a análise de variância, ajustando-se equações aos dados disponiveis (Cousens, 1988). No primeiro caso, avalia-se a densidade de infestação a partir da qual é significativa a redução de rendimento da cultura e, no segundo, determina-se o período durante o qual a presença da planta daninha é mais competitiva para a cultura e acarreta perdas de produção estatisticamente significativas (Caussanel et al., 1986; Zimdahl, 1980, 1993).

A existência desses períodos pressupõe que as plantas cultivadas têm uma fase durante o seu ciclo biológico mais sensivel à interferência, que corresponde ao período em que a cultura deve estar isenta de infestantes. Destaca-se que o NCA tem apenas componentes biológicos (relativos às plantas daninhas e cultivadas), o que conduz frequentemente a conclusões menos corretas (Cousens, 1988). A adição de componentes referentes ao tempo e à economia, por exemplo, pode melhorar as estimativas obtidas.

b. Nivel Prejudicial de Ataque (NPA). O NPA é chamado de seuil de dêgats economiques (Caussanel et al., 1986) ou economic injury level (Cousens, 1988; Coble \& Mortensen, 1992) em francês e inglês, respectivamente. Em matologia/herbologia, o NPA representa a densidade de infestação a partir da qual os gastos no controle das infestantes são menores que os prejuizos causados por elas. Segundo Coussans et al. (1986), esse nivel é aquele a que todos os outros devem-se reportar. Convém ressaltar que o NPA incorpora componentes biológicos e econômicos, o que não acontece com o NCA.

Com o NPA, tem-se por objetivo obter resposta à seguinte pergunta:

\section{- A partir de qual densidade de infestação é economicamente vantajoso controlar a(s) infestante(s)?}

O NPA representa uma análise de custo/ beneficio. De acordo com Aguiar (1992), para seu cálculo há necessidade de conhecer alguns componentes. Primeiramente, tem-se a função de competição (Y) para a combinação cultura-infestante considerada, de modo a prever o efeito da infestante na redução de produção da cultura. Em segundo lugar, tem-se a produção potencial paga ao produtor (Ypp), que corresponde à produção da cultura isenta de infestantes (Ycc) deduzidas as frações não pagas ao produtor devido, nomeadamente, ao efeito da infestante na qualidade da produção. Adicionalmente, deve-se conhecer também o preço unitário da produção $(\mathrm{P})$, o qual pode depender do efeito da infestante na qualidade da produção. Finalmente, é preciso conhecer a eficácia dos meios de controle no combate à infestante na cultura (E) e os seus custos (C).

Vale ressaltar que o impacto das infestantes sobre a cultura pode repercutir em vários aspectos do agroecossistema, de forma que esses efeitos devem ser dimensionados e quantificados economicamente-por exemplo, custos de meios de controle usados ou evitados no combate às pragas, resultantes do efeito da infestante na entomofauna: no primeiro caso, deduzem-se os custos dos meios de controle usados no combate à infestante e, no segundo, adicionam-se.

As estimativas de redução da produção dependem das espécies infestantes, de sua população e das culturas que estão convivendo com elas. Assim, a correta identificação das espécies daninhas e a estimativa exata da sua população são importantes para obter boas recomendações. Outros fatores também afetam o impacto das infestantes nas culturas. Por exemplo, quando as populações infestantes são elevadas, o acréscimo adicional de uns indivíduos pode não ter muito efeito. Isso se deve ao fato de que plantas começam a competir umas com as outras, além de competirem com a cultura.

Com vistas a facilitar as tomadas de decisão para gestão de plantas daninhas, sugerese documentar a previsão de perdas causadas por plantas sob baixas densidades populacionais (Vidal et al., 2004; Adati et al., 2006; NCSU, 2009), assumindo que as infestantes emergiram ao mesmo tempo que a cultura (NCSU, 2009). Normalmente, sob baixas densidades populacionais de plantas daninhas é que existe a dúvida sobre a real necessidade de empregar medidas de controle. 
Alternativamente, sob alta densidade de plantas daninhas, não há dúvidas quanto à necessidade de controle, mas é conveniente conhecer o potencial competitivo individual de cada espécie para decidir quais devem ser priorizadas no controle. Quando as densidades de plantas daninhas são muito elevadas, é impossivel obter contagens precisas de todas e cada uma das espécies daninhas. Para essas áreas, uma estimativa do número total de plantas daninhas pode ser suficiente, pois, à medida que o número de plantas aumenta, o efeito por indivíduo diminui. Nessas situações, o mais importante é definir a relação das populações de plantas daninhas para estimar com precisão o melhor tratamento possível de ser recomendado (NCSU, 2009).

Na determinação do NPA em pesquisas com uma única espécie vegetal, os modelos de competição frequentemente usados em herbologia podem ser classificados em linear e hiperbólico (Cousens, 1985), quando envolvem densidade de infestantes e rendimento da cultura, ou múltiplo linear e múltiplo não linear (Aguiar, 1992), quando envolvem o fator tempo de interferência (Tabela 1).

O NPA $\left(d^{*}\right)$ é, portanto, função de: $d^{*}=f(C$, $\mathrm{P}, \mathrm{QR}, \mathrm{E}, \mathrm{Ypp}$ ).

A eficácia dos meios de manejo das infestantes $(\mathrm{E})$ toma valores entre 0 e 1 e exprime a proporção de infestantes eliminadas, sendo calculada pela fórmula:

$\mathrm{E}=1-\frac{\text { densidade das infestantes após o controle }}{\text { densidade das infestantes antes do controle }}$

Partindo da definição, o NPA pode ser representado matematicamente pela equação 1 :

$$
\left.\mathrm{C}=\mathrm{P}\left[\mathrm{Ypw}\left[\mathrm{d}^{*}(1-\mathrm{E})\right]-\mathrm{Ypw}\left(\mathrm{d}^{*}\right)\right] \quad \text { (eq. } 1\right)
$$

em que Ypw $\left(d^{*}\right)$ representa a produção da cultura para a densidade (ou biomassa) $\mathrm{d}^{*}$, obtida pela aplicação da função de competição; e Ypw [d* $(1-E)]$ representa a produção da cultura para a densidade (ou biomassa) de infestantes que resulta da aplicação dos meios de controle $\left[\mathrm{d}^{*}(1-\mathrm{E})\right]$.

Na equação 1 estão incluídos pelo menos quatro pressupostos. Primeiro, os produtos utilizados no controle de infestantes não têm efeito negativo na cultura. Caso haja efeito
Tabela 1 - Modelos para determinação da relação entre a redução de produção da cultura com a densidade de infestação (linear e hiperbólico) e com o tempo de competição (múltiplo linear e não linear)

\begin{tabular}{|l|l|}
\hline \multicolumn{1}{|c|}{ Nome } & \multicolumn{1}{c|}{ Modelo } \\
\hline Linear & $\mathrm{QR}^{1 /}=\mathrm{a}+\mathrm{bd}$ \\
\hline Hiperbólico & $\mathrm{QR}=(\mathrm{Id}) /(1+((\mathrm{Id}) / \mathrm{A}))$ ou $\mathrm{QR}=(\mathrm{Ad}) /\left(\mathrm{d}_{1 / 2}+\mathrm{d}\right)$ \\
\hline Múltiplo linear & $\mathrm{QR}=\mathrm{a}+\mathrm{bd}+\mathrm{gt}+\mathrm{edt}$ \\
\hline
\end{tabular}

Múltiplo não linear $\mathrm{QR}=\mathrm{a}+\mathrm{bd}+\mathrm{cd}^{2}+\mathrm{gt}+\mathrm{ht}^{2}+\mathrm{edt}$

1/ QR representa a redução de rendimento da cultura; $d$ é a densidade de infestação, biomassa; $t$ representa o período de convivência; a, b, c, e, g, h, I, A, d 1/2 $_{2}$ representam os parâmetros das equações de ajuste.

deletério, ele pode ser calculado de acordo com Spader \& Vidal (2001) e Vidal et al. (2002). O segundo pressuposto é que, quando as infestantes são combatidas, ainda não ocorreu qualquer prejuízo na cultura. Terceiro, a cultura e a infestante considerada sofrem poucos efeitos da competição exercida por outras infestantes. Finalmente, os custos e beneficios dizem respeito apenas ao ano considerado.

\section{b.1. NPA a partir do modelo linear}

O modelo linear que relaciona a densidade de infestantes e a redução do rendimento da cultura é muito útil para baixas infestações. O modelo linear parte do pressuposto de que a perda de produção devido a uma infestante é constante e independente da densidade de infestação. Ele só deixa de ser válido apenas quando as densidades se elevam de forma que a área de abrangência de cada infestante começa a se sobrepor (Cousens, 1985). Sob baixa infestação de plantas daninhas há mais necessidade de determinar o NPA, pois é justamente nesta condição que se têm dúvidas na necessidade ou economicidade de medidas de controle das infestantes (Vidal et al., 2004; Adati et al., 2006; NCSU, 2009). Introduzindo na equação 1 o modelo linear apresentado na Tabela 1, tem-se:

$\mathrm{C}=\mathrm{P}\left\{\mathrm{Ypp}\left[1-\left(\mathrm{a}+\mathrm{bd}^{*}(1-\mathrm{E})\right)\right]-\mathrm{Ypp}\left[1-\left(\mathrm{a}+\mathrm{bd} \mathrm{d}^{*}\right)\right]\right\}$

Resolvendo a equação de forma a se obter a NPA $\left(d^{*}\right)$, chega-se à equação 2 :

$$
\mathrm{d}^{*}=\mathrm{c} /\left(\mathrm{b} E \mathrm{PY}_{\mathrm{pp}}\right)
$$




\section{b.2. NPA a partir do modelo hiperbólico}

O modelo hiperbólico é obtido quando se avalia o rendimento da cultura sob o efeito de ampla faixa de infestação, abrangendo desde baixas densidades até populações além da capacidade de suporte do meio. O modelo hiperbólico tem como pressuposto fundamental perdas nulas quando a densidade de infestação é zero e atinge o seu valor máximo, que nunca ultrapassa os $100 \%$, quando a densidade de infestação é muito elevada, exibindo comportamento linear para as densidades baixas (Cousens, 1987; Adati et al., 2006). Outro pressuposto do modelo hiperbólico é que em cada tratamento avaliado a densidade de plantas infestantes deve ser mantida fixa, do início do experimento até a colheita da cultura (Cousens, 1985; Aguiar, 1992). Introduzindo na equação 1 o modelo hiperbólico apresentado na Tabela 1, tem-se a equação 3 :

$C=P\left[Y p p\left[1-\frac{I^{*}(1-E)}{1+\frac{I^{*}(1-E)}{A}}\right]-Y p p\left[1-\frac{I^{*}}{1+\frac{I^{*}}{A}}\right]\right]$

Obtendo o NPA $\left(d^{*}\right)$ da equação 3 , tem-se:

$d^{*}=\frac{A\left[\sqrt{E} \sqrt{A^{2} E P^{2} Y p p^{2}+2 A C P Y p p(E-2)+C^{2} E}-A E P Y p p+C(2-E)\right]}{2 C I(E-1)}$

(eq. 4)

A solução da equação 4 é indeterminada quando $\mathrm{E}=1$. Alternativamente, para a situação específica em que $(1-E)$ equivale a zero, pode-se substituir na equação 3 este valor e obter a equação 5 :

$$
\mathrm{C}=\mathrm{P}\left[\mathrm{Ypp}-\mathrm{Ypp}\left[1-\frac{\mathrm{Id}^{*}}{1+\frac{\mathrm{Id}^{*}}{\mathrm{~A}}}\right]\right]
$$

Obtendo-se o NPA da equação 5, resulta em:

$$
\mathrm{d}^{*}=\frac{\mathrm{AC}}{\mathrm{I}(\mathrm{A} \mathrm{P} \mathrm{Ypp}-\mathrm{C})}
$$

Para facilitar o cálculo do NPA obtido a partir do modelo hiperbólico, é possivel utilizar a equação resultante da equação 6 , em que $(1-E)$ afeta todo o modelo e não apenas $\mathrm{d}^{*}$, como na equação 3 . Assim:

$$
\mathrm{C}=\mathrm{P}\left[\mathrm{Ypp}\left[1-\frac{\mathrm{Id}^{*}}{1+\frac{\mathrm{Id}^{*}}{\mathrm{~A}}}(1-\mathrm{E})\right]-\mathrm{Ypp}\left[1-\frac{\mathrm{Id}^{*}}{1+\frac{\mathrm{Id}^{*}}{\mathrm{~A}}}\right]\right]
$$

O NPA pode ser calculado pela equação 8:

$$
\mathrm{d}^{*}=\frac{\mathrm{AC}}{\mathrm{I}(\mathrm{A} \text { E P Ypp }-\mathrm{C})}
$$

\section{b.3. Modelo múltiplo linear}

O modelo múltiplo para a análise da relação entre densidade de infestantes e redução do rendimento leva em consideração a população e o tempo de convivência entre plantas daninhas e cultura. Introduzindo na equação 1 o modelo múltiplo simples apresentado na Tabela 1, obtém-se:

$$
\begin{aligned}
& \mathrm{C}=\mathrm{P}\left[\mathrm{Ypp}\left(1-\mathrm{a}-\mathrm{bd} d^{*}(1-\mathrm{E})-\mathrm{gt}-\mathrm{ed}^{*} \mathrm{t}(1-\mathrm{E})\right)-\right. \\
& \left.\mathrm{Ypp}\left(1-\mathrm{abd}^{*}-\mathrm{gt}-\mathrm{ed}^{*} \mathrm{t}\right)\right]
\end{aligned}
$$

A determinação do NPA, então, resulta na equação 10 :

$$
\mathrm{d}^{*}=\frac{\mathrm{C}}{\text { E P Ypp }(\mathrm{b}+\mathrm{et})}
$$

\section{b.4. Modelo múltiplo não linear}

Como no modelo anterior, este modelo envolve densidade de plantas e tempo na análise do rendimento da cultura. Inserindo na equação 1 o modelo múltiplo de competição não linear da Tabela 1, resulta na equação 11 :

$$
\begin{aligned}
& \mathrm{C}=\mathrm{P}\left[\mathrm { Ypp } \left(1-\mathrm{a}-\mathrm{bd} \mathrm{d}^{*}(1-\mathrm{E})-\mathrm{cd}^{* 2}-\mathrm{gt}^{\mathrm{h}}-\mathrm{ht}^{2}-\mathrm{ed}^{*} \mathrm{t}(1-\right.\right. \\
& \text { E) } \left.)-\mathrm{Ypp}\left(1-\mathrm{abd}^{*}-\mathrm{cd}^{* 2}-\mathrm{gt}-\mathrm{ht}^{2}-\mathrm{ed}^{*} \mathrm{t}\right)\right]
\end{aligned}
$$

O NPA é calculado por intermédio da fórmula:

$$
\mathrm{d}^{*}=\frac{\mathrm{C}}{\text { E P Ypp }(\mathrm{b}+\text { et })}
$$

É de notar que as equações 10 e 12 são iguais, razão pela qual o cálculo do NPA com 
os modelos múltiplos linear e não linear fazse com recurso à mesma fórmula. Esse fato deve-se à resolução das equações para determinar $\mathrm{d}^{*} \mathrm{e}$ implica a anulação de parâmetros associados ao tempo de competição, o que conduz à não utilização de toda a informação disponivel dos modelos múltiplos. Para evitar essa limitação e tirar maior proveito dos modelos múltiplos, propõe-se a equação 13 , com a qual, conhecendo os valores de C, P e Ypp, se determina a redução de produção tolerável $\left(\mathrm{Y}^{*}\right)$, valor a partir do qual é economicamente vantajoso controlar a infestante. Esse valor, aplicado aos gráficos de "curvas de nivel" elaborados a partir dos modelos múltiplos de competição, permite conhecer o período de tempo (número de dias) em que uma determinada densidade da infestante pode permanecer na cultura sem causar prejuízos superiores aos custos do seu controle.

$$
\mathrm{Y}^{*}=\frac{\mathrm{C}}{\mathrm{P} \text { Ypp E }} \times 100
$$

\section{c. Nivel Econômico de Ataque (intervenção)} (NEA). No Brasil este termo também é denominado nível de dano econômico (NDE). O NEA é conhecido na literatura francesa e na inglesa como seuil d'intervention (Caussanel et al., 1986) e economic threshold (Cousens, 1988; Coble \& Mortensen, 1992), respectivamente. O NEA é a intensidade de dano das infestantes sobre a cultura a partir da qual se devem aplicar medidas limitativas ou de controle para impedir que a cultura sofra prejuizos superiores ao custo das medidas a adotar, ou seja, para impedir que se atinja o NPA (Amaro \& Baggiolini, 1982; Mexia et al., 1994).

Na herbologia, os conceitos de NEA e NPA normalmente são considerados sinônimos. Isso ocorre porque, na maioria das situações, a duração de uma geração das plantas infestantes é muito superior ao tempo necessário para tomar a decisão de tratar ou não e à oportunidade do tratamento (Cousens, 1987; Mexia et al., 1994).

d. Nivel de Tolerância (NT). O NT no idioma francês é referido como seuil de tolérance (Caussanel et al., 1986). Esse conceito é aplicável ao nível econômico de ataque e ao nível prejudicial de ataque, no sentido em que se tolera a presença das infestantes das culturas até um nível economicamente confortável (Amaro \& Baggiolini, 1982). Ele pode traduzirse na seguinte questão:

- A partir de qual valor de redução de produção (\%) é economicamente vantajoso controlar a(s) infestante(s)?

O NT pode ser obtido a partir da equação 14 :

$$
\mathrm{Y}^{*}=\frac{\mathrm{C}}{\mathrm{P} \text { Ypp E }} \times 100
$$

Nota-se que cada densidade de infestação tem um período específico a partir do qual é economicamente vantajoso combater a infestante.

Pelo exposto, os cálculos de prejuízos com as plantas daninhas deverão ter por objetivo obter resposta à seguinte questão:

\section{- Quanto tempo pode permanecer uma dada densidade de infestação sem causar prejuizo?}

Para responder a esta pergunta, é necessário recorrer a modelos múltiplos de competição (Fernandes, 1994), cujos fatores independentes são a densidade da(s) infestante(s) e o tempo de competição.

e. Nivel de Segurança de Ataque (intervenção) (NSA). O NSA também é referido como seuil de securité (Caussanel et al., 1986) em francês. O nível de segurança resulta da aplicação de um coeficiente de segurança atribuído arbitrariamente ao NPA ou a qualquer outro nível de ataque (Coussans et al., 1986). O seu aparecimento tem a ver com a natural aversão dos agricultores ao risco e à eventual não verificação dos pressupostos dos vários niveis de ataque (Auld \& Tisdell, 1986).

Atendendo às características de arbitrariedade que encerram (por exemplo, densidades de infestantes fixas durante o período de estudo) e ao fato de os experimentos que se fazem para determinar a função de produção normalmente favorecerem a interferência das infestantes, os coeficientes de segurança poderiam ser dispensados. 


\section{INFESTAÇÃO COM PLANTAS DE DIFERENTES ESPÉCIES VEGETAIS}

Nas condições reais de campo, as infestações são constituídas por várias espécies vegetais. Nesse caso, há necessidade de outra abordagem para quantificar os NPA, NEA e NDE. Durante o período crítico de interferência das plantas daninhas na cultura, o número de plantas infestantes é determinado por espécie. O método de cálculo desenvolvido a seguir tem como base o modelo linear. Como apresentado anteriormente, eles são mais bem empregados em situações de baixa densidade populacional da cada espécie (Vidal et al., 2004; Adati et al., 2006; NCSU, 2009).

Quando a avaliação é realizada ao final do ciclo das plantas daninhas, pode-se coletá-las para determinar sua massa e o rendimento de grãos por unidade de área. Modelos de regressão linear múltipla determinam a fração relativa à interferência inter e intraespecífica (equação 15).

$$
1 / \mathrm{W}=\mathrm{b}_{\mathrm{co}}+\mathrm{b}_{\mathrm{cc}} \mathrm{N}_{\mathrm{c}}+\mathrm{b}_{\mathrm{cw} 1} \mathrm{~N}_{\mathrm{w} 1}+\ldots+\mathrm{b}_{\mathrm{cwi}} \mathrm{N}_{\mathrm{wi}}
$$

em que $\mathrm{W}$ é a biomassa ou o rendimento por planta da cultura; $b_{c o}$ é a interseção da equação de regressão; $b_{c c}$ é o coeficiente de interferência intraespecífica da cultura; $\mathrm{N}_{\mathrm{c}}$ é a densidade da cultura; $b_{c w 1}$ é o parâmetro de interferência interespecífica entre a cultura e a espécie 1; $\mathrm{N}_{\mathrm{w} 1}$ é a densidade da espécie $1 ; \mathrm{b}_{\mathrm{cwi}}$ é o parâmetro de interferência interespecífica entre a cultura e a espécie $\mathrm{i}$; e $\mathrm{N}_{\mathrm{wi}}$ é a densidade da espécie i. Pela equação 15, a espécie infestante é considerada efetiva quando seu coeficiente de interferência interespecífico for significativo estatisticamente. Para determinar o nivel de dano econômico, utiliza-se o procedimento descrito por Swinton et al. (1994). Os dados de rendimento de grãos de trigo e a densidade efetiva de infestantes serão ajustados ao modelo da hipérbole retangular apresentado na equação 16, para obter as estimativas dos

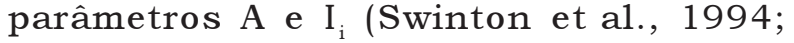
Gherekhloo et al., 2009).

$$
Y=Y_{w f}\left[1-\frac{\sum I_{i} w_{i}}{100\left(1+\frac{\sum I_{i} w_{i}}{A}\right)}\right]
$$

em que Y é a estimativa do rendimento da cultura; $\mathrm{Y}_{\mathrm{wf}}$ é o rendimento da cultura nas parcelas sem interferência de plantas infestantes; $I_{i}$ representa a porcentagem de perda do rendimento associada com a espécie $i ; w_{i}$ é a densidade da espécie i; e o parâmetro A representa a assintota da máxima porcentagem de perda do rendimento. $O$ parâmetro $I_{i}$ pode ser convertido no valor do índice competitivo relacionado à carga competitiva total (TCL) por meio da divisão pelo maior parâmetro $I_{\mathrm{i} \text {. }}$ (aquele da espécie mais competitiva), como descrito pela equação 17 :

$$
T C L=w_{1}+\frac{I_{2}}{I_{1}} w_{2}+\frac{I_{3}}{I_{1}} w_{3}+\ldots+\frac{I n}{I_{1}} w n
$$

em que $w_{1}$ é a densidade da espécie mais competitiva; $I_{1}$ é a porcentagem de perda do rendimento associada com a infestante 1 (a mais competitiva); e os parâmetros $\mathrm{I}_{2}, \mathrm{w}_{2}, \mathrm{I}_{3}$, $\mathrm{w}_{3}, \mathrm{I}_{\mathrm{n}}, \mathrm{w}_{\mathrm{n}}$ são os coeficientes das demais espécies infestantes (desde que significativas estatisticamente). A porcentagem de perda do rendimento é calculada pela equação 18 :

$$
Y L(\%)=\frac{\left(Y_{w f}-Y\right)}{Y_{w f}} \times 100
$$

em que $\mathrm{Y}_{w f}$ é estimado pela equação 16. Utilizando os valores de TCL no lugar de densidade no modelo da hipérbole retangular, novos parâmetros A e I serão calculados, como visto na equação 19:

$$
Y L(\%)=\frac{I(T C L)}{1+\frac{I(T C L)}{A}}
$$

Utilizando os novos A e I (estimados pela equação 19), o nível de dano econômico é calculado conforme a proposta de O'Donovan (1991), dada pela equação 20:

$$
D=\frac{1-\left[\frac{Y P-H}{Y P}\right]}{\frac{I}{100}+\frac{I}{A}\left[\frac{Y P-H}{Y P}-1\right]}
$$

em que $\mathrm{D}$ é o nível de dano econômico baseado na carga competitiva total (TCL); Y, o rendimento de grãos da cultura nas parcelas sem 
interferência de infestantes $\left(\mathrm{kg} \mathrm{ha}^{-1}\right)$; $\mathrm{P}$, o preço dos grãos; e H, o custo do controle.

\section{CONSIDERAÇÕES FINAIS}

A introdução na década de 1980 dos modelos matemáticos nos estudos das relações competitivas entre culturas e plantas daninhas veio melhorar a compreensão do fenômeno da interferência e permitir o cálculo de niveis de prejuízo entre ambos os grupos de plantas (Aguiar, 1992). A utilização de modelos de regressão entre densidade e perdas do rendimento passa a ser recomendada em detrimento dos testes de comparação de média, devido aos resultados pouco fiáveis destes em relação àqueles (Cousens, 1988). O uso dos modelos através de técnicas de regressão é uma forma rigorosa de analisar os dados, que conduz a conclusões úteis e corretas do ponto de vista agronômico (Kropff \& Spitters, 1991; Coble \& Mortensen, 1992; Swinton et al., 1994; McDonald \& Riha, 1999).

Os modelos apresentados nesta revisão são denominados empíricos, descritivos ou de regressão. Existem, no entanto, outros modelos, denominados explanatórios, mecanicistas ou dinâmicos, que se baseiam nos processos fisiológicos envolvidos na interferência e que dão melhores níveis de explicação para esse fenômeno que os primeiros ou apresentam relação de causa e efeito. No entanto, a limitada utilidade prática desses modelos (Kropff \& van Laar, 1993) e os elevados meios materiais exigidos os tornam pouco atrativos.

A utilização prática dos modelos empíricos tem sido possível após formação de amplo banco de dados de simples obtenção (Mexia, 1985). Os modelos matemáticos, obtidos através de métodos de regressão, permitem a integração dos componentes biológicos e econômicos; assim, é possivel fazer os cálculos dos diferentes niveis de prejuízos por meio de programas computacionais, como o HADSS (Herbicide Application Decision Support System) (NCSU, 2009), obtendo-se os beneficios preconizados pelo modelo de proteção integrada (Mexia, 1985; Fernandes, 1994, 2003). Esses modelos possibilitam, ainda, a determinação das perdas de produção em situações não experimentadas e, dessa forma, orienta pesquisas futuras (Caussanel et al., 1986).

\section{AGRADECIMENTOS}

Ao CNPQ, pelo apoio ao segundo autor.

\section{LITERATURA CITADA}

\begin{abstract}
ADATI, C.; OLIVEIRA, V. A.; KARAM, D. Análise matemática e biológica dos modelos de estimativa de perdas de rendimento na cultura devido à interferência de plantas daninhas. Planta Daninha, v. 24, n. 1, p. 1-12, 2006.
\end{abstract}

AGOSTINETTO, D. et al. Dano econômico como critério na decisão sobre manejo de genótipos de arroz concorrentes em arroz irrigado. Pesq. Agropec. Bras., v. 40, n. 1, p. 1-9, 2005.

AMARO, P.; BAGGIOLINI, M. (Eds.). Introdução à protecção integrada. Roma: FAO/DGPPA, 1982. 276 p.

AGUIAR, C. F. G. Estudos herbológicos no trigo em Trásos-Montes numa óptica de protecção integrada. 1992 183 f. Dissertação (Mestrado em Proteção Integrada) Universidade Técnica de Lisboa, Lisboa, 1992.

AULD, B. A.; TISDELL, C. A. Economic threshold/critical density models in weed control. In: EWRS SYMPOSIUM WEED CONTROL, 1986, Brighton. Proceedings...

Brighton: 1986. p. 261-268.

BENTO, F. M.; FERNANDES, J. D.; JANSEN, H. G. Biologia e concorrência da milhã-pé-de-galo (Echinochloa crus-galli (L.) Beauv. e erva-moira (Solanum nigrum L.) na cultura do tomateiro. Prot. Prod. Agric., v. 1, p. 75-91, 1987.

BIANCHI, M. A.; FLECK, N. G.; LAMEGO, F. P. Proporção entre plantas de soja e plantas competidoras e as relações de interferência mútua. Ci. Rural, v. 36, n. 5, p. $1380-1387,2006$

CAUSSANEL, J. P. et al. La variation des seuils de nuisibilité des mauvaises herbes Resultats experimentaux. Persp. Agric. v. 109 , n. 1 , p. $22-27,1986$

COBLE, H. D.; MORTENSEN, D. A. The threshold concept and its application to weed science. Weed Technol., v. 6, n. 1, p. 191-195, 1992.

COUSENS, R. A simple model relating yield loss to weed density. Ann. Appl. Biol., v. 107, p. 239-252, 1985.

COUSENS, R. Theory and reality of weed control thresholds Plant Prot. Quart., v. 2, n. 1, p. 19-20, 1987.

COUSENS, R. Misinterpretation of results in weed research through inappropriate use of statistics. Weed Res., v. 28, n. 4, p. 281-289, 1988.

COUSSANS, G. W.; COUSENS, R. D.; WILSON, B. J. Thresholds for weed control - The concepts and their interpretation. In: EWRS SYMPOSIUM WEED CONTROL, 1986, Brighton. Proceedings... Brighton: 1986. p. 253-260. 
FERNANDES, J. M. P. V. Estudos de competição da ervamoira na cultura do tomate para indústria numa perspectiva de protecção integrada. $1994.137 \mathrm{f}$. Dissertação (Mestrado em Proteção Integrada) - Universidade Técnica de Lisboa, Lisboa, 1994.

FERNANDES, J. M. P. V. Ecologia da flora espontânea e competição da erva-moira (Solanum nigrum l.) na cultura do tomate para indústria. 2003. $225 \mathrm{f}$. Tese (Doutorado em Engenharia Agronômica) - Universidade Técnica de Lisboa, Lisboa, 2003

FLECK, N. G. Interferência de papuã (Brachiaria plantaginea) com soja e ganho de produtividade obtido através do seu controle. Pesq. Agropec. Gaúcha, v. 2, n. 1, p. 63-68, 1996.

FLECK, N. G.; RIZZARDI, M. A.; AGOSTINETTO, D. Nível do dano econômico como critério para tomada de decisão no controle de guanxuma em soja. Planta Daninha, v. 20, n. 3 , p. $421-429,2002$

GALON, L. et al. Níveis de dano econômico para decisão de controle de capim-arroz (Echinochloa spp.) em arroz irrigado (Oryza sativa). Planta Daninha, v. 25, n. 4, p. 709-718, 2007.

GHEREKHLOO, J. et al. Evaluation of multispecies weed competition and their economic threshold on wheat crop using regression equations. Planta Daninha, 2009. (Submited)

GODINHO, I.; COSTA, J. C. A. Concorrência da Phalaris minor Retz na cultura do trigo. In: CONGRESSO NACIONAL DE HERBOLOGIA, 3., 1980, Lisboa. Anais... Lisboa: 1980. p. 237-243.

KROPFF, M. J.; SPITTERS, C. J. T. A simple model of crop loss by weed competition from early observations on relative leaf area of weeds. Weed Res., v. 31, n. 1, p. 97-105, 1991.

KROPFF, M. J.; van LAAR, H. H. Modelling crop-weed interactions. Manila: CAB/IRRI. 1993. 274 p. Disponível em: $<$ http://books.irri.org/0851987451_content.pdf>. Acesso em: 29 maio 2009

McDONALD, A. J.; RIHA, S. J. Model of crop: weed competition applied to maize: Abutilon theophrasti interactions II. Assessing the impact of climate: implications for economic thresholds. Weed Res., v. 39, n. 5, p. 371-381, 1999.

MEXIA, A. Os prejuízos causados pelas infestantes nas culturas. Cad. Soc. Fitiatria Fitofarmacol., v. 2, n. 1, p. 1-57, 1985.

MEXIA, A.; MOREIRA, I.; AGUIAR, C. Nível económico de ataque e conceitos afins em herbologia. In: ENCONTRO NACIONAL DE PROTECÇÃO INTEGRADA, 2., 1994, Vila Real. Anais... Vila Real: Universidade de Trás-os-Montes e Alto Douro, 1994. p. 397-400.
NORTH CAROLINA STATE UNIVERSITY - NCSU. WEBHADSS population help. Disponível: $<\mathrm{http}$ :// www.webhadss.ncsu.edu/help/Help.asp?Title=Weed $\% 20$ Population\%20Help\&HelpFile=/Help/ WeedsPresandpop.txt>. Acesso em: 29 maio 2009.

O'DONOVAN, J. T.; BLACKSHAW, R. E. Effect of volunteer barley (Hordeum vulgare L.) interference on field pea (Pisum sativum L.) yield and profitability. Weed Sci., v. 45 , n. 2, p. $249-255,1997$

PASSINI, T.; CHRISTOFFOLETI, P. J.; YADA, I. F. U. Competitivity of the common-bean plant relative to the weed alexandergrass [Brachiaria plantaginea (Link) Hitch.]. Sci. Agric., v. 60, n. 2, p. 259-268, 2003.

RIZZARDI, M. A.; FLECK, N. G.; AGOSTINETTO, D. Nível de dano econômico como critério para controle de picão-preto em soja. Planta Daninha, v. 21, n. 2, p. $273-282,2003$.

RIZZARDI, M. A. et al. Interferência de populações de Euphorbia heterophylla e Ipomoea ramosissima isoladas ou em misturas sobre a cultura da soja. Planta Daninha, v. 22, n. 1, p. 29-34, 2004.

SPADER, V.; VIDAL, R. A. Seletividade e dose de injúria econômica de nicosulfuron aplicado em diferentes estádios de desenvolvimento da cultura do milho. Ci. Rural, v. 31, n. 6, p. 929-934, 2001.

SWINTON, S. M. et al. Estimation of crop yield loss due to interference by multiple weed species. Weed Sci., v. 42, n. 1, p. 103-109, 1994.

THEISEN, G.; VIDAL, R. A.; FLECK, N. G. Redução da infestação de Brachiaria plantaginea em soja pela cobertura do solo com palha de aveia-preta. Pesq. Agropec. Bras., v. 35, n. 4 , p. $753-756,2000$.

VIDAL, R. A. et al. G. Análise comparativa da interferência de poáceas na cultura do milho nos EUA e no Brasil. R. Bras. Agroci., v. 5, n. 3, p. 182-184, 1999.

VIDAL, R. A. et al. Dose de injúria econômica do herbicida cyanazine na cultura do milho. R. Bras. Herbic., v. 2, n. 2/3, p. 127-132, 2002.

VIDAL, R. A. et al. Nível do dano econômico de Brachiaria plantaginea na cultura de milho irrigado. Planta Daninha, v. 22, n. 1, p. 63-69, 2004.

ZIMDAHL, R. L. Weed crop competition: a review. Oregon: International Plant Protection Center/Oregon State University, 1980. 195 p.

ZIMDAHL, R. L. Fundamentals of weed science. San Diego: Academic Press, 1993. 450 p. 